\title{
Combination of Adaptive Modulation and Power Management for the Performance Enhancement of MIMO-OFDM Systems
}

\author{
Sheng Hong* ${ }^{(1)}$, Hanzhong Chunyu ${ }^{(1)}$, Kefei Liu ${ }^{(1)}$ and Manos Tentzeris ${ }^{(2)}$ \\ (1) School of Electronics and Information Engineering, Beihang University, \\ Beijing, 100083, China \\ (2) School of Electrical and Computer Engineering, Georgia Institute of \\ Technology, Atlanta, GA 30332-0250,USA \\ E-mail: fengqiao1981@gmail.com
}

\begin{abstract}
Adaptive modulation and power management has recently been considered as an efficient means to boost communication performance in MIMO-OFDM system. In this work, a new scheme of joint modulation and power management is investigated. It adopts the implementation of a Signal-to-Interference-Noise-Ratio threshold and MQAM-MPSK hybrid modulation structure for every parallel subchannel. The simulation result displays that this approach efficiently achieves a lower BER in contrast to other adaptive modulation schemes with the same Eb/N0, as both 16QAM and QPSK.
\end{abstract}

Index Terms -OFDM, MIMO, Adaptive modulation, Power management, SINR

\section{Introduction}

As the service demand of wireless communications is growing rapidly, high speed and high reliability become critical factors for the systems' design, requiring an ever increasing number of frequency bands and power levels. Nevertheless, the radio spectrum available for wireless communication and the dynamic power range for the portable terminals are extremely limited. MIMO and OFDM are the main technologies of $4^{\text {th }}$ generation systems, which have a strong diversity processing ability and a high frequency efficiency. In order to further promote the performance of MIMO-OFDM system, this paper provides a new strategy using a Signal-to-Interference-Noise-Ratio threshold and an MQAM-MPSK modulation hybrid structure for every parallel subchannel. It saves more power and gets better BER in contrast to adaptive modulations, such as 16QAM and QPSK.

\section{Design Concept and Structure}

A multicarrier system can be efficiently implemented in discrete time using an inverse FFT (IFFT) to act as a modulator and an FFT to act as a demodulator. The transmitted data are the "frequency" domain coefficients and the samples at the output of the IFFT stage are "time" domain samples of the transmitted waveform. Fig.1 shows a typical MIMO-OFDM implementation [1]. 

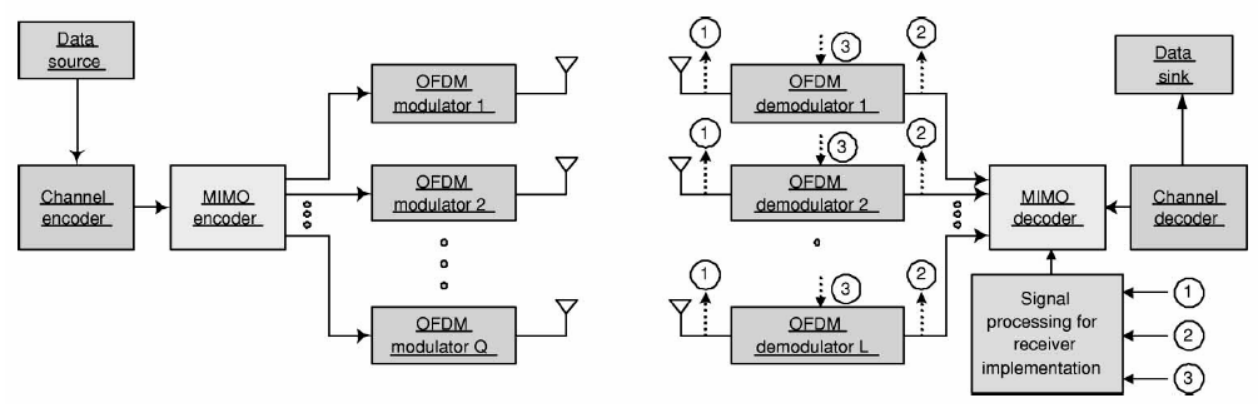

Fig.1 Q $\times$ L MIMO-OFDM system, where $\mathrm{Q}$ and $\mathrm{L}$ are the numbers of inputs and outputs, respectively.

In this paper, we focus on adopting a Signal-to-Interference-Noise-Ratio threshold and a MQAM-MPSK hybrid modulation structure for every parallel subchannel, upon which we implement an adaptive modulation and power management. Fig.2 illustrates a specific function diagram.

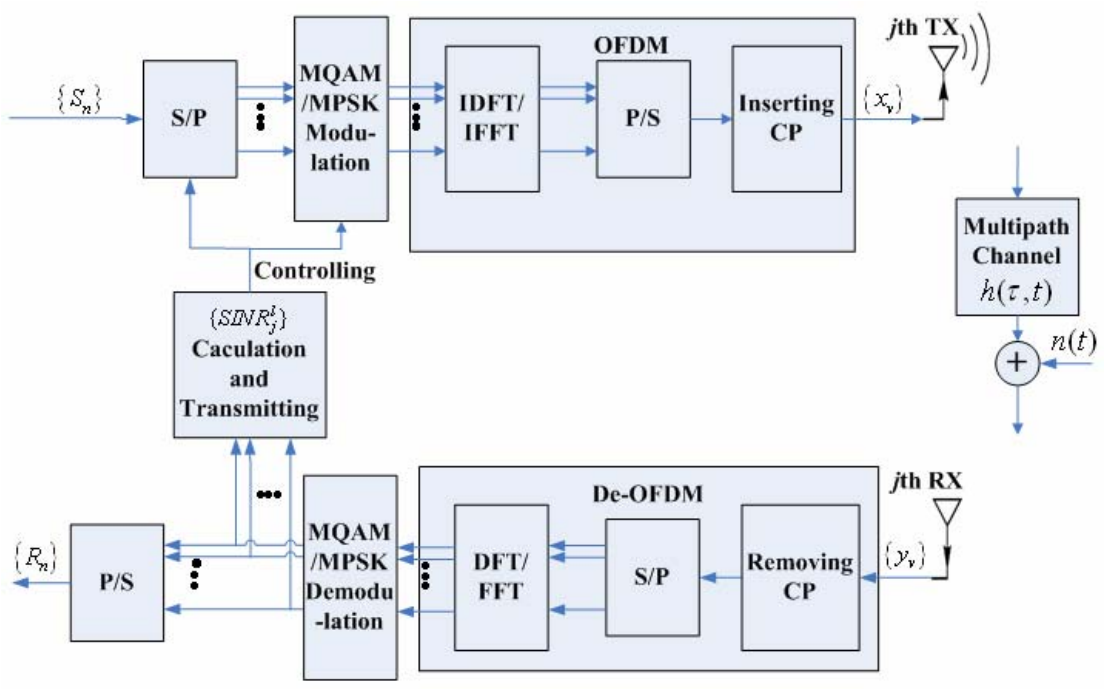

Fig.2 Function block diagram of joint adaptive management system.

Reference to [2], we migrate the signal description from user expression into subchannel expression as follows. For a specific communication link, the sampled signal on the $l$-th subchannel of the $j$-th TX $-j$-th RX link can be expressed as:

$$
x_{j}^{l}(n)=\sum_{k=1}^{K} \sqrt{P_{k}^{l} G_{k j}^{l}} s_{k}^{l}(n)+n_{j}^{l}(n)
$$

where $P_{k}^{l}$ and $G_{k j}^{l}$ is the transmitted power and propagation loss from the $k$-th TX to the $j$-th RX in the $l$-th sub-channel, respectively, $s_{k}^{l}(n)$ is message symbol from the $k$-th TX to the $j$-th $\mathrm{RX}$ at time $\mathrm{n}$, and $n_{j}^{l}(n)$ is the sampled thermal noise. We assume that the each subchannel are in the sate of flat fading. Without loss of generality, we assume $N_{j}^{l}=N_{0}$. The $j$-th RX's SINR at subchannel $l$ can be expressed as:

$$
\operatorname{SINR}_{j}^{l}=\frac{P_{j}^{l} G_{j j}^{l}}{\sum_{k \neq j} P_{k}^{l} G_{k j}^{l}+N_{0}}
$$


We assume the channel conditions of different subcarriers for each TX/RX are assumed perfectly estimated. There exists reliable feedback channels from RX to TX without any delay so that $\operatorname{SINR}_{j}^{l}$ can be fed back instantly. Moreover for a practical mobile system, the OFDM frequency offset between the TX and the RX is around several tens of $\mathrm{Hz}$. The intercarrier interference caused by the frequency offset may cause some error floor increase. However, this is not the bottleneck limiting the system performance and this offset can be fed back to the mobile terminal for adjustment. Meantime, one guard interval is put in front of IDFT/IFFT symbol such that multipath fading can be minimized and synchronized algorithm is applicable for each subcarrier. So in this paper, we assume TX and RX are synchronized.

We calculate the $\operatorname{SINR}_{j}^{l}$ of the $l$-th subchannel in the $j$-th $\mathrm{RX}$ from (2), and then feed it back to the $j$-th TX. The $l$-th subchannel in the $j$-th TX adopts different modulation means in terms of different received $\operatorname{SINR}_{j}^{l}$. In this paper, we choose three kind of modulation means as simulation cases such as 4QAM, 8PSK and 16QAM owing to their easily implemented and used frequently in real environment. The selection of the modulation means can be described by the following function (3), where $\alpha$ and $\beta$ are the two SINR thresholds selected based on the channel situation in the range of which the wireless system regularly operates. By this scheme, each subchannel in TX can change its modulation and control its power independently.

$$
f\left(\operatorname{SINR}_{j}^{l}\right)=\left\{\begin{array}{l}
4 Q A M, \quad \operatorname{SINR}_{j}^{l} \leq \alpha \\
8 P S K, \quad \alpha<\operatorname{SINR}_{j}^{l} \leq \beta \\
16 Q A M, \quad \operatorname{SINR}_{j}^{l}>\beta
\end{array}\right.
$$

\section{Simulation Results}

We evaluate the performance of the proposed scheme by choosing a communication link of TX/RX for a MIMO-OFDM system. The OFDM symbol has 128 subcarriers and covers $3.2 \mathrm{MHz}$ bandwidth. Each OFDM symbol occupies $50 \mu \mathrm{S}$ time interval and 20k block rate. The wireless channel has the multipath Rayleigh fading part and Additive White Gaussian Noise part. The maximum power is Pmax $=50 \mathrm{mWatts}$, and the thermal noise level is $N_{0}=10^{-4}$ Watts. The specific parameters are listed in Table 1.

Table 1: The setup of system parameters

\begin{tabular}{|c|c|c|c|c|c|}
\hline $\begin{array}{c}\text { Guard } \\
\text { Interval }\end{array}$ & $10 \mu \mathrm{S}$ & $\begin{array}{c}\text { Cyclic Prefix } \\
\text { Length Lg }\end{array}$ & 10 samples & $\begin{array}{c}\text { Sub-channel } \\
\text { Interval } \Delta \mathrm{f}\end{array}$ & $25 \mathrm{KHz}$ \\
\hline $\begin{array}{c}\text { IDFT/IFFT } \\
\text { Period T TFT }\end{array}$ & $40 \mu \mathrm{S}$ & $\begin{array}{c}\text { Modulation } \\
\text { Means }\end{array}$ & 4QAM/8PSK/16QAM & $\begin{array}{c}\text { OFDM Symbol } \\
\text { Bandwidth }\end{array}$ & $3.2 \mathrm{MHz}$ \\
\hline $\begin{array}{c}\text { OFDM Symbol } \\
\text { Period T }\end{array}$ & $50 \mu \mathrm{S}$ & $\begin{array}{c}\text { Subcarrier } \\
\text { Number }\end{array}$ & 128 & $\begin{array}{c}\text { Sampling } \\
\text { Frequency }\end{array}$ & $1 \mathrm{MHz}$ \\
\hline
\end{tabular}

To evaluate the performance of the new approach, we have tested $10^{5}$ sets of 
frequency selective fading channels, which are simulated using one four-ray Rayleigh model [3] with an exponential power profile and 100ns root-meansquare (RMS) delay spread.

As shown in Fig.3, the proposed algorithm shows a better performance than 16QAM and QPSK in MIMO-OFDM system. When $\mathrm{Eb} / \mathrm{N} 0$ is equal to $12 \mathrm{db}$, the adaptive modulation and power management algorithm achieves a BER value lower than $10^{-5}$. Also, the proposed algorithm gets at most $7 \mathrm{~dB}$ processing gain compared with 16QAM and $0.4 \mathrm{~dB}$ processing gain compared with QPSK when they are at the same BER close to $10^{-5}$.

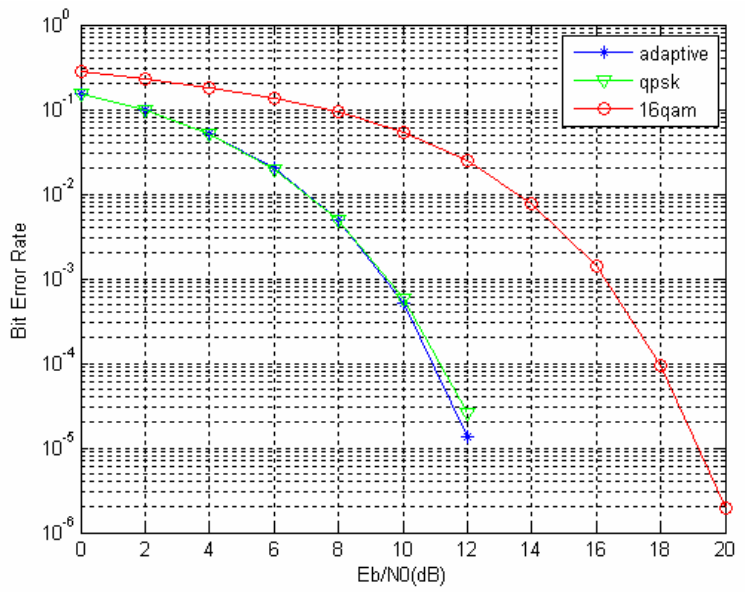

Fig.3 BER vs. Eb/N0

\section{Discussion and Conclusions}

This paper presents a novel adaptive modulation and power management algorithm for MIMO-OFDM systems. It adopts a Signal-to-Interference-Noise-Ratio threshold and MQAM-MPSK hybrid modulation structure for every subchannel. The simulation results demonstrated that the proposed approach features the lowest BER compared with regular 16QAM and QPSK in MIMO-OFDM systems with the same $\mathrm{Eb} / \mathrm{N} 0$. In other words, it gets a higher processing gain and saves more power while maintaining the same BER.

\section{Acknowledgment}

This work was supported in part by the China Scholarship Council, P. R. China.

\section{References:}

[1] Gordon 1. Stuber, John R. Barry, Steve W. Mclaughlin, Ye(Geoggerey) Li, Mary Ann Ingram, Thomas G. Pratt. "Broadband MIMO-OFDM Wireless Communications". Proceedings of The IEEE, vol. 92, pp.271-294, Feb 2004.

[2] Zhu Han, Zhu Ji, and K. J. Ray Liu. "Power Minimization for Multi-Cell OFDM Networks Using Distributed Non-cooperative Game Approach". IEEE Communications Society, Globecom 2004.

[3] T. S. Rappaport, Wireless communications, Prentice Hall, 1996. 